\title{
Cost-Effectiveness Analysis of Linagliptin in Japan Based on Results from the Asian Subpopulation in the CARMELINA ${ }^{\circledR}$ Trial
}

\author{
Hirotaka Watada · Hiroyuki Sakamaki · Daisuke Yabe · \\ Fumiko Yamamoto - Tatsunori Murata - Keigo Hanada • \\ Tetsuaki Hirase · Tomoo Okamura
}

Received: May 19, 2020 / Published online: June 17, 2020

(C) The Author(s) 2020

\section{ABSTRACT}

Introduction: We evaluated the cost-effectiveness of linagliptin in Japan by estimating the lifetime outcome based on clinical event rates from the Asian subpopulation of the CARMELINA trial. In CARMELINA, linagliptin added to standard of care (SoC) versus SoC demonstrated noninferiority with regard to risk of composite

Digital Features To view digital features for this article go to https://doi.org/10.6084/m9.figshare.12388196.

Electronic Supplementary Material The online version of this article (https://doi.org/10.1007/s13300020-00852-8) contains supplementary material, which is available to authorized users.

H. Watada $(\bowtie)$

Department of Metabolism and Endocrinology, Juntendo University Graduate School of Medicine, Tokyo, Japan

e-mail: hwatada@juntendo.ac.jp

H. Sakamaki $(\bowtie)$

Graduate School of Health Innovation, Kanagawa University of Human Services, Kanagawa, Japan

e-mail: hiroyuki.sakamaki@nifty.com

D. Yabe

Department of Diabetes and Endocrinology, Gifu

University Graduate School of Medicine, Gifu, Japan

D. Yabe

Yutaka Seino Distinguished Center for Diabetes Research, Kansai Electric Power Medical Research Institute, Kobe, Japan cardiovascular (CV) outcome in patients with type 2 diabetes at high risk of $\mathrm{CV}$ and kidney events. Issues resulting from conducting a costeffectiveness analysis using data from a clinical noninferiority study were also investigated.

Methods: A microsimulation model was used to evaluate linagliptin/SoC versus SoC in terms of direct costs and quality-adjusted life years (QALYs) from a Japanese public healthcare payer's perspective. Cost data were obtained from recent Japanese publications. The time horizon was defined as lifetime, and the discount rate for costs and effectiveness was $2 \%$ per year. One-way and probabilistic sensitivity analyses were performed.

\section{Yabe}

Division of Molecular and Metabolic Medicine, Kobe University Graduate School of Medicine, Kobe, Japan

F. Yamamoto $\cdot$ T. Okamura

Nippon Boehringer Ingelheim Co., Ltd., Tokyo, Japan

T. Murata $\cdot$ K. Hanada

CRECON Medical Assessment Inc, Tokyo, Japan

T. Hirase

Eli Lilly Japan K.K., Kobe, Japan 
Results: In the base case analysis, and taking medical history into account, the incremental effectiveness of linagliptin/SoC versus SoC was 1.34 QALYs, and the incremental cost for linagliptin was $-545,319$ yen. In the one-way sensitivity analysis, the parameter which most affected the results was the hazard ratio for renal failure of linagliptin/SoC compared with SoC. The probabilistic sensitivity analysis showed that the probability of reduced costs and increased effectiveness (dominant) was $48 \%$. Assuming an incremental cost-effectiveness ratio (ICER) threshold of 5 million yen, the probability that the ICER was below the threshold was $89 \%$ for linagliptin/SoC compared with SoC.

Conclusions: This evaluation, using Asian subpopulation data from the CARMELINA trial, suggested that the cost-effectiveness of linagliptin for a lifetime outcome was favourable in Japan. However, the results must be interpreted cautiously because of the noninferiority trial data source, which might cause ICER variations for each parameter.

Keywords: Cardiorenal events; CARMELINA trial; Cost-effectiveness analysis; Diabetes; DPP4 inhibitor; ICER; Japan; Linagliptin; MACE; QALY

\section{Key Summary Points}

\section{Why carry out this study?}

Anti-glycaemic therapies aim to prevent microvascular and macrovascular complications to increase life expectancy and improve quality of life. To this end, cost-effectiveness analysis using practical outcome data on antidiabetic drugs offers valuable information.

The CARMELINA trial was a long-term clinical study of the dipeptidyl peptidase 4 (DPP4) inhibitor linagliptin in patients with type 2 diabetes that confirmed the noninferiority of linagliptin plus standard of care (SoC) versus SoC for 3-point major adverse cardiovascular events.
Data from the Asian subpopulation of the CARMELINA trial were considered appropriate to evaluate the costeffectiveness of linagliptin added to SoC in patients with type 2 diabetes in Japan with estimated lifetime outcomes. The present analysis also investigated uncertainty resulting from conducting cost-effectiveness analysis with data from a noninferiority study.

\section{What was learned from this study?}

Overall, the results were favourable for linagliptin plus SoC, but the possibility of large incremental cost-effectiveness ratio (ICER) variations for each parameter resulting from the noninferiority trial data source mean that the data must be interpreted with caution.

The probabilistic sensitivity analysis showed that the probability of reduced costs and increased effectiveness (dominant) was $48 \%$.

Assuming an ICER threshold of 5 million yen, the probability that the ICER was below the threshold was $89 \%$ for linagliptin plus SoC compared with SoC.

\section{INTRODUCTION}

Of the microvascular and macrovascular complications associated with diabetes [1], cardiovascular (CV) diseases are of particular importance. The risk of developing $\mathrm{CV}$ diseases is two- to threefold that of people without diabetes [2] and CV death is a major cause of mortality in Japanese patients with diabetes [3]. The ultimate goal of anti-glycaemic therapy for diabetes is to prevent microvascular and macrovascular complications to increase life expectancy and quality of life (QoL) and to reduce medical costs $[4,5]$. Therefore, it is important to include diabetic complications in cost-effectiveness analyses to estimate lifetime outcomes in diabetes.

Dipeptidyl peptidase 4 (DPP4) inhibitors are administered to approximately $70 \%$ of patients 
receiving diabetes treatment in Japan and, in recent years, have become the first-line drug in Japanese patients with type 2 diabetes (T2D) [6]. The CARMELINA trial [7] was a long-term clinical study of the DPP4 inhibitor linagliptin in adult patients with T2D who were at high risk of $\mathrm{CV}$ and kidney events, and confirmed the noninferiority of linagliptin versus placebo for the primary endpoint (3-point major adverse $\mathrm{CV}$ event [3P-MACE]) when added to standard of care (SoC) [7].

The Asian subpopulation of the CARMELINA trial included 555 Asian patients living in Asia who were evaluated for their risk of $\mathrm{CV}$ and kidney events [8]. Data from the subgroup analysis of the CARMELINA trial were considered appropriate to perform a cost-effectiveness analysis in Japan using clinical CV and kidney outcome data, despite the fact that the analysis of the Asian subpopulation was a subgroup analysis and the sample size was not powered to detect statistical significance for the effect on each endpoint.

An economic model was developed, which extrapolated the outcomes of patients receiving linagliptin plus SoC compared with SoC in the Japanese clinical setting over the patients' remaining lifetime. The availability of quantitative outcome data allows this model to rely exclusively on observed event rates, rather than estimating event rates from extrapolated changes in biomarker values (e.g. glycated haemoglobin).

The objective of the present analysis was (1) to evaluate the cost-effectiveness of linagliptin added to SoC in Japan with estimated lifetime outcomes from the perspective of a public healthcare payer, using data from the CARMELINA Asian subgroup analysis in patients with T2D, and (2) to investigate issues, including uncertainty, resulting from conducting a costeffectiveness analysis using the data from a noninferiority study.

\section{METHODS}

This article is based on previously conducted studies and does not contain any studies with human participants or animals performed by any of the authors.

\section{Model Structure and Analytical Methods}

Using TreeAge Pro Version 2019 R2.1 (Boston, MA, USA), a microsimulation model was developed and a base case analysis was performed to assess the cost-effectiveness of linagliptin plus SoC compared with SoC, as per the two patient groups included in the CARMELINA trial (ClinicalTrials.gov NCT01897532) [7, 8].

The six-step simulation process using the microsimulation model is shown in Fig. 1. The microsimulation model was used to simulate the process of repeated experiences of multiple events. Microsimulation tracks patients at an individual level and therefore can take into account the impact of each individual medical history and time spent; additionally, using endpoint data from an outcomes trial, the microsimulation model allows estimation of cost-effectiveness beyond the trial follow-up period to a lifetime horizon. According to the International Society for Pharmacoeconomics and Outcomes Research-Society for Medical Decision Making modeling task force, a microsimulation model enables the estimation of the long-term impact of intervention for people with T2D [9]. Furthermore, the United Kingdom Prospective Diabetes Study (UKPDS) regression model, which is widely used for costeffectiveness analysis for antidiabetic drugs, was also constructed on the basis of a microsimulation model [10]. An analysis cycle of 1 month was chosen to minimise the influence of competing risks occurring when multiple events were simultaneously considered and qualityadjusted life years (QALYs) were used as an effectiveness measure. The time horizon was defined as a lifetime. Only direct medical costs were included and the discount rate for costs and effectiveness was $2 \%$ per year. The analyses were conducted according to current Japanese guidelines for cost-effectiveness evaluations [11].

\section{Modelling Cohort}

The present analysis used data from the Asian subpopulation $(n=555)$ included in the CARMELINA trial [7, 8]; patients were from Japan, 


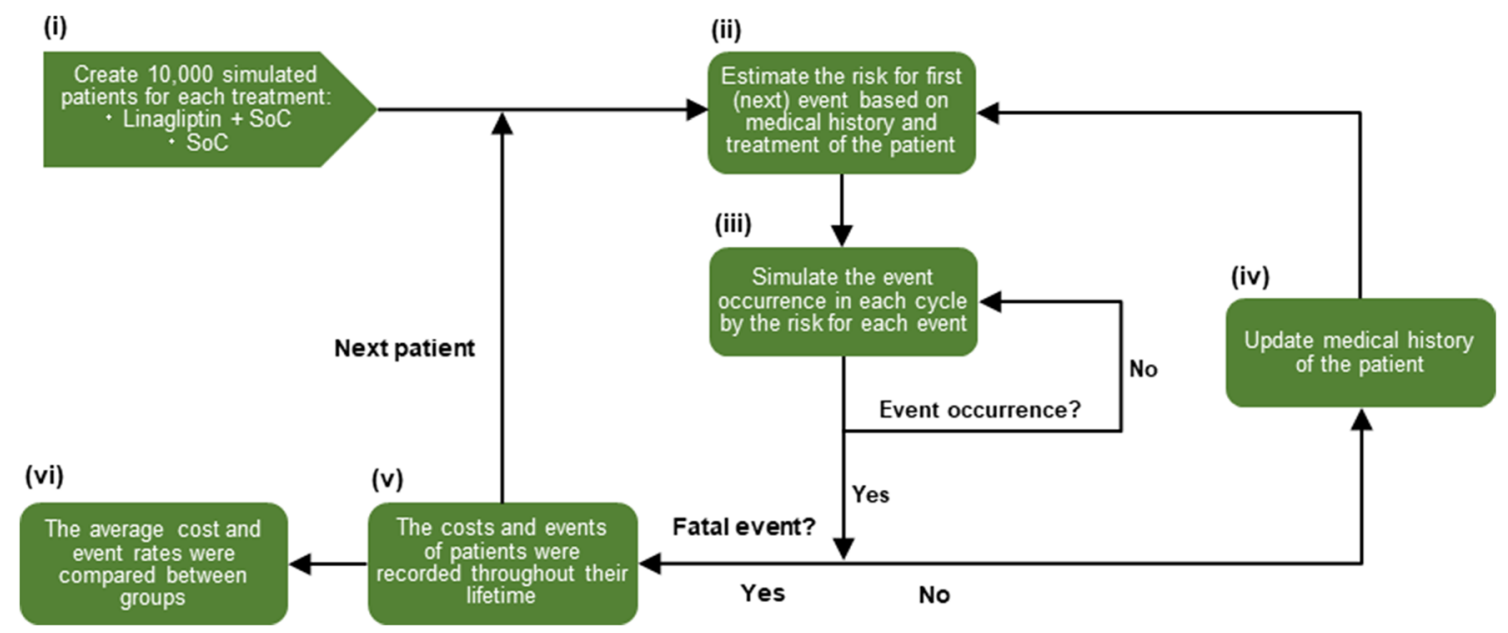

Fig. 1 Microsimulation flow. (i) 10,000 simulated patients were created for each treatment. (ii) The incidence rate of each event (nonfatal myocardial infarction, nonfatal stroke, hospitalisation due to unstable angina, hospitalisation due to heart failure, albuminuria progression, renal failure, and CV death) was estimated, according to the medical history and treatment of each patient, on the basis of the results from the CARMELINA Asian subpopulation analysis. (iii) The occurrence/non-occurrence of an

China, South Korea, Taiwan, and Malaysia, and self-identified as being of Asian race [8]. During a 2.2-year median follow-up period, linagliptin treatment had a neutral cardiorenal profile and was not found to be associated with 3P-MACE in Asian patients, with a similar event occurrence rate in linagliptin plus SoC- and SoCtreated patients $(10.7 \%$ and $11.7 \%$, respectively; hazard ratio [HR] $0.90 ; 95 \%$ confidence intervals [CI] 0.55-1.48). Linagliptin was associated with a nominal decrease in the risk of hospitalisation for heart failure (HR 0.47; 95\% CI 0.24-0.95) [8].

\section{Parameters}

\section{Clinical Parameters}

The mean age of the Asian subpopulation in the CARMELINA study used for the present analysis was 65 years. The incidence of events included in this analysis (nonfatal myocardial infarction, nonfatal stroke, hospitalisation due to unstable angina, hospitalisation due to heart failure, albuminuria progression, renal failure, and CV event in each cycle was simulated on the basis of the risk for each event. (iv) The medical history of each patient was updated. Processes (ii)-(iv) were repeated until a fatal event occurred in the simulated patients. (v) The costs and events of each simulated patient were recorded throughout their lifetime. (vi) The average cost and event rates were compared between the linagliptin plus SoC group and SoC group. $C V$ cardiovascular, $\mathrm{So} C$ standard of care

death) was reported as event incidence per month. This was calculated from the events observed in the analysis of the Asian subgroup of the CARMELINA trial [8] using the personyear (PY) method.

To estimate the risk for each event, the baseline event risk was estimated from the data of the SoC group using data from the CARMELINA Asian subgroup analysis (Table 1). The event risk for linagliptin was estimated by multiplying the event risk calculated for the SoC group by the HR observed for the linagliptin/SoC group in the CARMELINA Asian subgroup analysis.

Additionally, this analysis attempted to evaluate how the risk of subsequent CV or kidney events would be increased in the lifetime simulation. To estimate the effect of medical history on the risk of a subsequent $\mathrm{CV}$ or kidney event, the regression coefficient, which was estimated from a previously published costeffectiveness analysis in Japan using data from another Asian subgroup analysis of a CV outcome trial [12], was converted to an HR for use in this analysis. Supplementary Table S1 
Table 1 Event risk setting

\begin{tabular}{|c|c|c|}
\hline \multirow[t]{2}{*}{ Event risk } & \multicolumn{2}{|l|}{ Values } \\
\hline & Event risk of SoC (event number/PY) & HR for linagliptin plus SoC (95\% CI) \\
\hline Nonfatal myocardial infarction & $19 / 608.1$ & $0.87(0.45,1.69)$ \\
\hline Nonfatal stroke & $10 / 610.9$ & $0.60(0.22,1.66)$ \\
\hline Hospitalisation for unstable angina & $3 / 614.3$ & $0.68(0.11,4.07)$ \\
\hline Hospitalisation for heart failure & $23 / 596.3$ & $0.47(0.24,0.95)$ \\
\hline Albuminuria progression & $63 / 245.7$ & $0.95(0.66,1.36)$ \\
\hline Renal failure & $14 / 612.5$ & $0.58(0.24,1.39)$ \\
\hline CV death & $13 / 622.5$ & $0.70(0.30,1.64)$ \\
\hline
\end{tabular}

$C I$ confidence interval, $C V$ cardiovascular, $H R$ hazard ratio, $P Y$ person-year, $S o C$ standard of care

presents the assessment of the increased risk of an event due to a medical history of events included in this analysis.

\section{Cost Parameters}

The present analysis was performed from the perspective of the public healthcare payer, using only direct medical costs, including patient copayments (reported in yen). The drug cost of linagliptin was set according to the National Health Insurance Drug Price List at the time of analysis, and the treatment costs for each event were obtained from a previous study [12].

The treatment costs for each event were estimated on the basis of the results calculated from data in the EBM Provider ${ }^{\circledR}$-a Japanese Claims database provided by Medical Data Vision Co., Ltd.-in a previously published costeffectiveness analysis in Japan [12] (Supplementary Table $\mathrm{S} 2$ ). For the costs associated with renal failure, only dialysis was considered. This analysis did not take into account the impact of the medical payment system revision rate that was implemented every other year in Japan because the difference in the medical payment rate between that used in this analysis [12] and the 2018 implementation was only $+0.55 \%$, and the impact on the results was negligible. The cost of SoC was not considered because SoC treatment was received by both groups in the CARMELINA trial.

\section{Utility Parameters}

The QALYs of linagliptin added to SoC or SoC alone were estimated by subtracting the disutility of the occurrence of each event during the time horizon from the QALYs calculated by accumulating baseline utility weights of patients included in the analysis. The baseline utility weight and the disutility at the occurrence of each event were obtained from a previously published analysis using the EuroQol 5-dimension 3-level (EQ-5D-3L) measure of health value in an American civilian population [13]. This study did not include the decrease in utility with hospitalisation for unstable angina and therefore this was assumed to be the same as that for nonfatal myocardial infarction (Supplementary Table S2).

\section{Sensitivity Analysis}

The impacts of uncertainty and variability around the model inputs were tested using a one-way sensitivity analysis series and a probabilistic sensitivity analysis. To evaluate the magnitude of the effect of each parameter on the analysis results, a one-way sensitivity analysis was performed. To display the results of the one-way sensitivity analysis pictorially, a tornado diagram was constructed using each parameter. The range of change in each parameter was $0-4 \%$ for the discount rate and $\pm 20 \%$ of default values for other parameters with the associated 95\% CI, when available. 
In addition, a probabilistic sensitivity analysis was performed with 10,000 Monte Carlo simulations in 10,000 patients to evaluate the uncertainty of the results. The stochastic parameters and utility parameters were assumed to have a beta distribution, the ratio data (HR) were assumed to be logarithmically normally distributed, and the cost parameters were assumed to have a gamma distribution [14].

A sensitivity analysis was conducted on the base case analysis to consider the effects of increased event risk due to medical history, as these factors will result in a conservative estimate for linagliptin.

\section{Scenario Analysis}

To evaluate the impact of using the point estimates of HR data, which were not tested for statistical significance, a scenario analysis was performed in cases where the HR of linagliptin on each event was set to 1.0 and where the costs for renal failure were assumed to be 0 yen.

A scenario analysis was conducted on the base case analysis considering medical history for the same reasons as the sensitivity analysis.

\section{RESULTS}

\section{Base Case Analysis According to Event Risk Increase due to Medical History}

This analysis aimed to evaluate how the risk of the subsequent $\mathrm{CV}$ and renal events would be increased, causing multiple events in the lifetime simulation. Accounting for effects of the increased risk of related events due to medical histories, the expected QALYs per study patient was 7.09 QALYs for linagliptin plus SoC and 5.75 QALYs for SoC. Hence, the incremental effectiveness of linagliptin was expected to be 1.34 QALYs (Table 2). The cost per study patient was $14,192,428$ yen for linagliptin/SoC and $14,737,747$ yen for SoC, leading to an incremental cost for linagliptin of - 545,319 yen.

Conversely, when the effects of increased risk of related events due to medical histories were not considered (Table 2), the expected QALYs per study patient was 10.26 QALYs for linagliptin plus SoC and 9.57 QALYs for SoC, resulting in an expected incremental effectiveness for linagliptin of 0.69 QALYs. The cost per study patient was $10,257,312$ yen for linagliptin plus SoC and 14,304,557 yen for SoC, and the incremental cost for linagliptin was - 4,047,246 yen.

In Table 3 and Fig. $2 a, b$, the cost analysis showing costs for each medical event in the two treatment groups is presented with and without consideration of the increased event risk due to medical history. The cost associated with renal failure (cost of dialysis) accounted for a large proportion of the estimated lifetime cost. The cost of dialysis was $11,238,312$ yen for linagliptin plus SoC and 11,409,876 yen for SoC when the effects of increased risk due to medical histories were considered. In comparison, when the effects of increased risk due to medical histories were not considered, the cost for renal failure (dialysis cost) was $8,316,517$ yen for linagliptin plus SoC and 12,638,256 yen for SoC.

\section{Sensitivity Analyses}

The one-way sensitivity analysis (Supplementary Table S3) demonstrated that the parameter which most affected the analysis results was the HR for renal failure of linagliptin plus SoC compared with SoC (Fig. 3). The probabilistic sensitivity analysis showed that the probability of increased costs and increased effectiveness was $43 \%$, and the probability of reduced costs and increased effectiveness (dominant) was $48 \%$ (Fig. 4). In addition, the probability that the gained QALYs adopted a negative value was $9 \%$, and the probability of increased costs was $43 \%$. In the case where the increased cost of linagliptin was excluded, the probability of increased costs was $41 \%$. Assuming an incremental cost-effectiveness ratio (ICER) threshold of 5 million yen [15], the probability that the ICER was below the threshold was $89 \%$ for the linagliptin plus SoC group compared with the SoC group.

\section{Scenario Analyses}

The ICER of linagliptin plus SoC compared with SoC was 3,481,897 yen/QALY. This increased 
Table 2 Base case analysis when the effects of increased risk of related events due to medical histories were/were not considered

\section{Lifetime cost (yen) Incremental costs (yen) QALYs QALYs gained ICER (yen/QALYs)}

Medical history considered

\begin{tabular}{llllll} 
SoC & $14,737,747$ & - & 5.75 & - & - \\
Linagliptin plus SoC & $14,192,428$ & $-545,319$ & 7.09 & 1.34 & Dominant \\
Medical history not considered & & & & \\
SoC & $14,304,557$ & - & 9.57 & - & - \\
Linagliptin plus SoC & $10,257,312$ & $-4,047,246$ & 10.26 & 0.69 & Dominant \\
\hline
\end{tabular}

$I C E R$ incremental cost-effectiveness ratio, $Q A L Y s$ quality-adjusted life years, $S o C$ standard of care

Table 3 Breakdown of costs considering/not considering the increased risk due to medical history

\begin{tabular}{|c|c|c|}
\hline & \multicolumn{2}{|l|}{ Cost (yen) } \\
\hline & $\overline{\text { SoC }}$ & Linagliptin plus SoC \\
\hline \multicolumn{3}{|l|}{ Medical history considered } \\
\hline Drug $\cos \mathrm{t}^{\mathrm{a}}$ & NA & 567,107 \\
\hline CV death & $1,129,847$ & 938,361 \\
\hline Renal failure & $11,409,876$ & $11,238,312$ \\
\hline Albuminuria progression & 0 & 0 \\
\hline Hospitalisation for heart failure & $1,655,472$ & 966,711 \\
\hline Hospitalisation for unstable angina & 29,807 & 30,254 \\
\hline Nonfatal stroke & 331,431 & 259,619 \\
\hline Nonfatal myocardial infarction & 181,314 & 192,064 \\
\hline \multicolumn{3}{|l|}{ Medical history not considered } \\
\hline Drug $\cos ^{\mathrm{a}}$ & NA & 815,879 \\
\hline CV death & 469,480 & 349,250 \\
\hline Renal failure & $12,638,256$ & $8,316,517$ \\
\hline Albuminuria progression & 0 & 0 \\
\hline Hospitalisation for heart failure & 556,624 & 284,082 \\
\hline Hospitalisation for unstable angina & 48,600 & 38,007 \\
\hline Nonfatal stroke & 310,023 & 196,019 \\
\hline Nonfatal myocardial infarction & 281,574 & 257,558 \\
\hline
\end{tabular}

$C V$ cardiovascular, $N A$ not applicable, $S o C$ standard of care

a 2019 linagliptin prices were used 


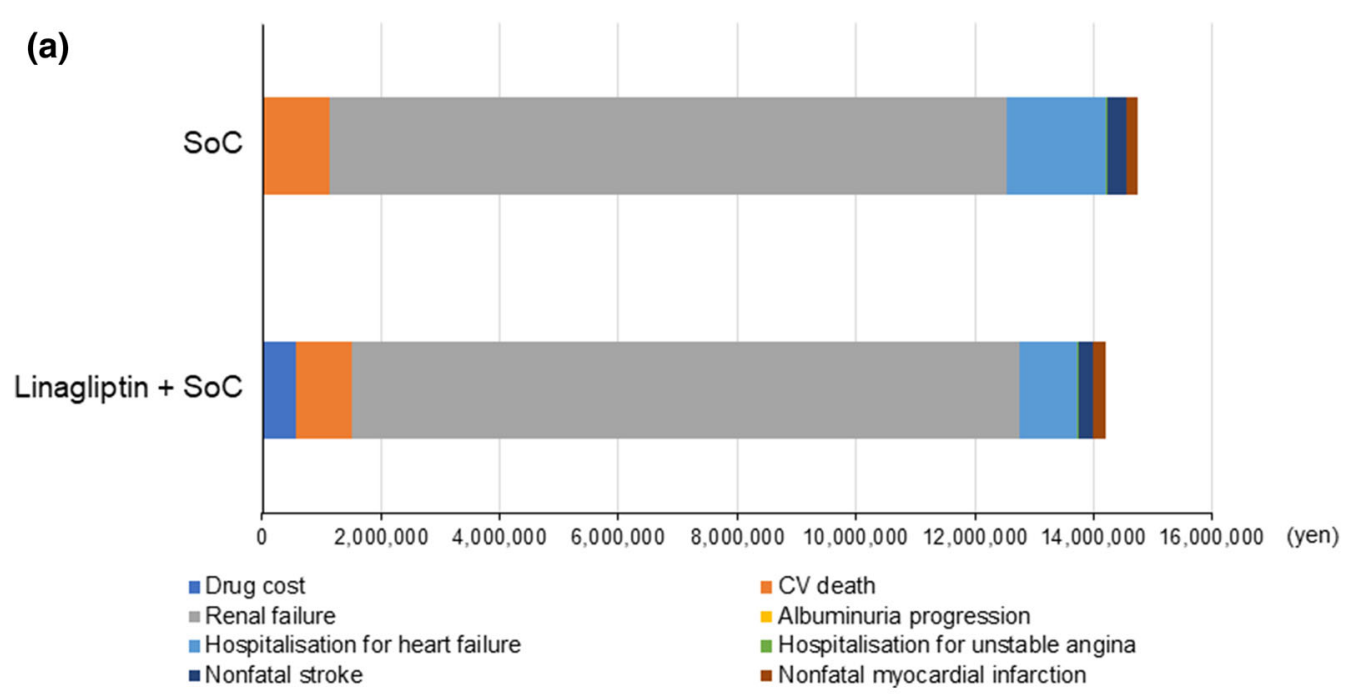

(b)

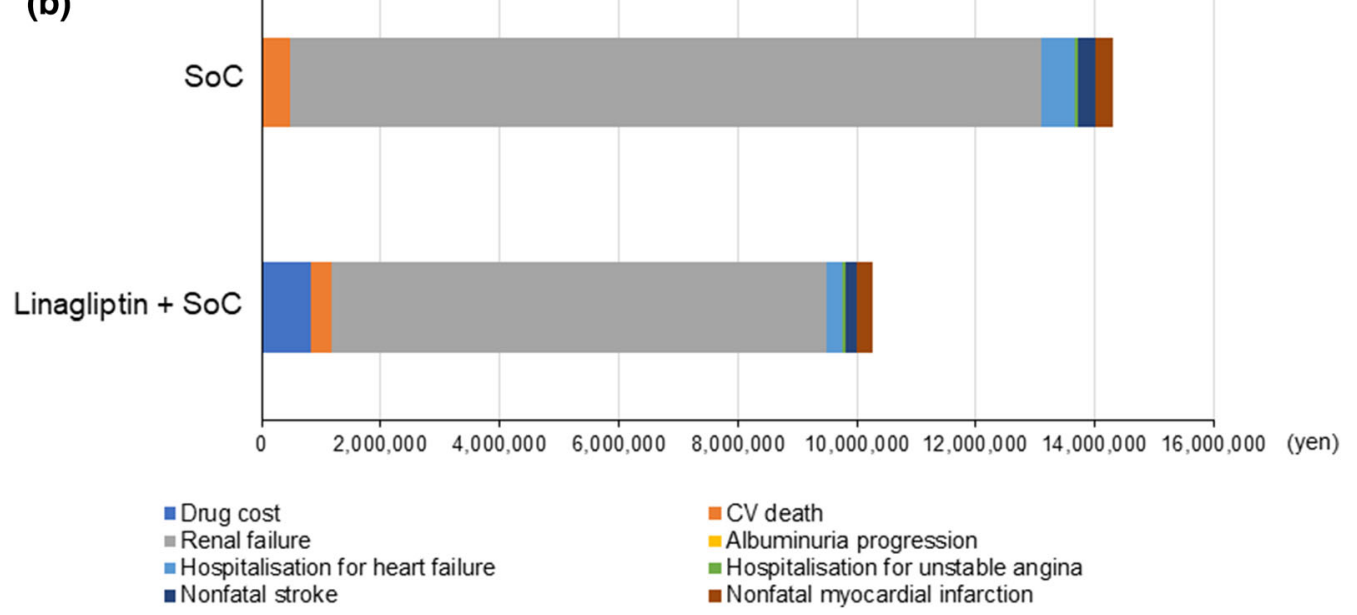

Fig. 2 Breakdown of costs considering (a) and not considering (b) the increased risk due to medical history. $C V$ cardiovascular, $S o C$ standard of care

when analysed with the HR for renal failure of linagliptin plus SoC set as 1.0 (Table 4).

\section{DISCUSSION}

The present study is a cost-effectiveness analysis for $\mathrm{CV}$ and renal event risk associated with treatment with linagliptin in addition to SoC in patients with T2D, based on the Asian subpopulation in the CARMELINA trial [8]. The use of data from a clinical noninferiority trial to perform a T2D cost-effectiveness study has previously been reported; an evaluation of medical resource usage associated with sitagliptin plus SoC versus SoC in the USA was based on the TECOS noninferiority trial [16].

We conducted two scenarios in the base case analysis, in which the first considered the effects of increased event risk due to medical history which might lead to a conservative estimate for linagliptin, while the second did not consider these factors. In both base case analyses using only point estimates of the CARMELINA trial results, treatment with linagliptin plus SoC was dominant compared with treatment with SoC. The difference in estimated costs between linagliptin plus SoC and SoC 


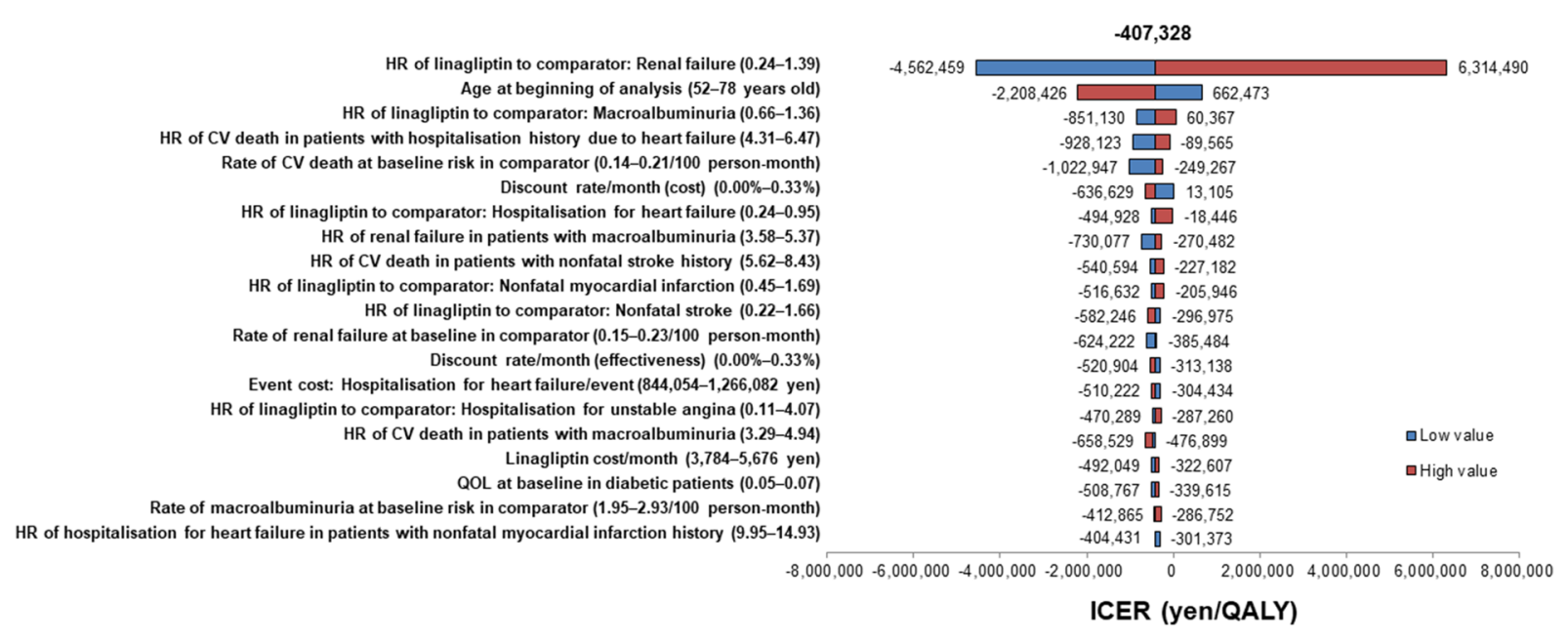

Fig. 3 One-way sensitivity analysis. The central vertical line in this tornado diagram represents the base case value. The blue bar shows the result when the parameter used a high value, and the red bar when changed to a low value.
The comparator was SoC. Renal failure had the greatest effect on the results of the analysis. $\mathrm{CV}$ cardiovascular, $H R$ hazard ratio, ICER incremental cost-effectiveness ratio, $Q A L Y$ quality-adjusted life year, $S o C$ standard of care

\section{Incremental QALY}

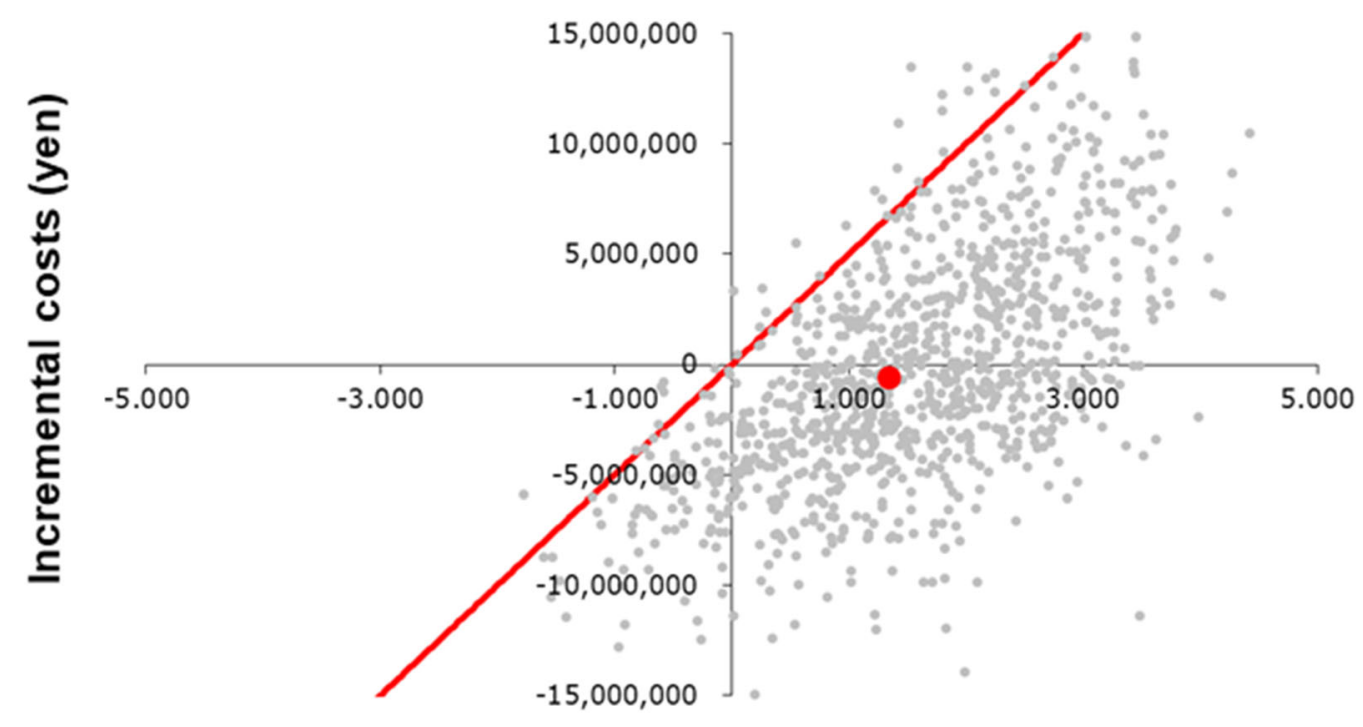

— ICER $=5$ million yen/QALY $\bullet$ ICER in base case analysis

Fig. 4 Probabilistic sensitivity analysis. The red line shows the reference value of the ICER in Japan set at 5 million yen/QALY. The red dot shows the ICER in the base case analysis. The probability of linagliptin plus SoC being considered as cost-effective (ICER $<5$ million yen) was $89 \%$ compared with the SoC group. ICER incremental cost-effectiveness ratio, $Q A L Y$ quality-adjusted life year, SoC standard of care 
Table 4 Scenario analysis

\section{Lifetime cost (yen) Incremental costs (yen) QALYs QALYs gained ICER (yen/QALYs)}

When the HR of linagliptin for nonfatal myocardial infarction was set to 1.0

$\begin{array}{llllll}\text { SoC } & 14,737,747 & - & 5.75 & - & - \\ \text { Linagliptin plus SoC } & 14,256,418 & -481,329 & 7.08 & 1.33 & \text { Dominant }\end{array}$

When the HR of linagliptin for nonfatal stroke was set to 1.0

$\begin{array}{llllll}\text { SoC } & 14,737,747 & - & 5.75 & - & - \\ \text { Linagliptin plus SoC } & 14,119,075 & -618,672 & 7.01 & 1.26 & \text { Dominant }\end{array}$

When the HR of linagliptin for hospitalisation for unstable angina was set to 1.0

$\begin{array}{llllll}\text { SoC } & 14,737,747 & - & 5.75 & - & - \\ \text { Linagliptin plus SoC } & 14,214,917 & -522,829 & 7.08 & 1.33 & \text { Dominant }\end{array}$

When the HR of linagliptin for hospitalisation for heart failure was set to 1.0

$\begin{array}{llllll}\text { SoC } & 14,737,747 & - & 5.75 & - & - \\ \text { Linagliptin plus SoC } & 14,763,956 & 26,209 & 6.91 & 1.16 & 22,521\end{array}$

When the HR of linagliptin for macroalbuminuria was set to 1.0

$\begin{array}{llllll}\text { SoC } & 14,737,747 & - & 5.75 & - & - \\ \text { Linagliptin plus SoC } & 14,267,341 & -470,406 & 7.06 & 1.31 & \text { Dominant }\end{array}$

When the HR of linagliptin for renal failure was set to 1.0

$\begin{array}{llllll}\text { SoC } & 14,737,747 & - & 5.75 & - & - \\ \text { Linagliptin plus SoC } & 19,132,619 & 4,394,872 & 7.01 & 1.26 & 3,481,897\end{array}$

When the HR of linagliptin for CV death was set to 1.0

$\begin{array}{llllll}\text { SoC } & 14,737,747 & - & 5.75 & - & - \\ \text { Linagliptin plus SoC } & 10,975,501 & -3,762,245 & 6.08 & 0.34 & \text { Dominant } \\ \text { Cost of renal failure }= & 0 & & & & - \\ \text { SoC } & 3,327,871 & - & 5.75 & - & \text { Dominant } \\ \text { Linagliptin plus SoC } & 2,954,116 & -373,754 & 7.09 & 1.34 & \text {. }\end{array}$

$C V$ cardiovascular, $H R$ hazard ratio, ICER incremental cost-effectiveness ratio, QALYs quality-adjusted life years, SoC standard of care

when considering the impact of increased risk of subsequent $\mathrm{CV}$ and kidney events in the lifetime simulation was smaller than the one without considering them. This could be attributable to the increased life years, which led to the increase in the contribution of renal failure, including dialysis, to the medical costs estimated with linagliptin plus SoC.
Of note, the CARMELINA Asian subpopulation analysis showed a nominally reduced risk of hospitalisation for heart failure when linagliptin was added to SoC [8]. This cost-saving feature could be one factor offsetting linagliptin treatment costs.

Previous cost-effectiveness analyses, in the USA and Europe, have commonly used a model 
where the development of diabetic complications is predicted on the basis of the UKPDS [10] to analyse cost-effectiveness over the lifetime horizon in diabetes, as measured by the ICER [17]. It is important to note that the model used in this study predicts clinical event rates exclusively using hard outcomes data from the CARMELINA clinical trial Asian subpopulation, without reliance on surrogate biomarkers. More commonly, diabetes models assume a cluster of major CV risk factors (e.g. high glycated haemoglobin, elevated blood pressure) and predispose determinants to predict $\mathrm{CV}$ risk in T2D. Treatment effects are then represented as changes in these surrogate markers with a set of risk equations, as in the UKPDS regression model [18], which is then used to predict treatment effect on CV events. This approach, however, is not adequate for an analysis of CV event risk based on the CARMELINA data, since calculations based on surrogate markers and UKPDS equations will not be able to capture the change in $\mathrm{CV}$ event rates observed in the CARMELINA clinical trial.

In Japan, the reference value of ICER for drug price adjustment has been set at 5 million yen [15]. As the probability that the ICER was below the threshold was $89 \%$ when the ICER threshold was assumed to be 5 million yen/QALY, the cost-effectiveness analysis of linagliptin in this analysis was considered to be appropriate even though it was based on a noninferiority study.

As shown in the scatter plot of the probabilistic sensitivity analysis, the probability for the gained QALYs to be a negative value was $9 \%$, the probability of reduced costs and increased effectiveness (dominant) was 48\% and the probability of increased costs and increased effectiveness was $43 \%$. If the increased drug cost of linagliptin was excluded, the probability of increased costs was $41 \%$; this is consistent with the results of noninferiority for the primary endpoint in both the overall population and the Asian subpopulation of the CARMELINA study $[7,8]$.

Previous reports have commented on the challenges of economic evaluations based on noninferiority trials and the uncertainty around the ICER [19]. The one-way sensitivity analysis conducted in this study showed that the HR for renal failure of linagliptin plus SoC compared with SoC had the greatest effect on the ICER, ranging from $-4,562,459$ yen to $6,314,490$ yen.

Taken together, the data suggest that the point estimates of each effectiveness parameter in this analysis were "favourable" using a noninferiority study; furthermore, in the probabilistic sensitivity analysis including overall parameters, the cost-effectiveness tended to appear favourable as a whole. However, as noted, the ICER range may fluctuate considerably in one-way sensitivity analyses; hence, the favourable cost-effectiveness outcome from this study should be interpreted with caution.

On the basis of the results of the group receiving SoC, this study showed that the treatment of renal failure makes a large contribution to the estimated medical costs when patients with T2D with a high CV and renal risk receive SoC. From a health economics perspective, it is significant that the current analysis revealed high medical costs for renal failure in patients with T2D in Japan, given that Japanese patients with T2D are more susceptible to diabetic nephropathy leading to end-stage renal failure compared with Caucasian patients $[20,21]$.

This study has some limitations that must be considered when interpreting the results. First, data from the assessment of the QoL of patients with diabetes in the USA, based on a European QoL measurement scale (the EQ-5D) [13], were used as utility scores, which were not assessed in Japan. Second, the data used to estimate the effect of medical history on the risk of a subsequent related $\mathrm{CV}$ and kidney event were obtained from another study analysing the costeffectiveness for patients with a high CV risk, directly based on Asian CV outcome data [12]. As the study population in this previous costeffectiveness publication was different from that of the CARMELINA study, the validity of the extrapolated data is limited. However, the extrapolation was attempted in order to estimate lifetime outcome after the follow-up period of the CARMELINA study, to assess the effects of increased event risk due to medical history on the analysis results. Third, for the patient population used in this analysis, the results were based on the data of those self- 
identifying as Asians in the CARMELINA study $[7,8]$ and were not obtained exclusively from Japanese patients. The patient population also included patients with relatively advanced diabetes with a high CV and renal risk; thus, the results may not be generalisable to the wider Japanese population with T2D. Finally, the CARMELINA trial demonstrated a noninferior risk of a composite $\mathrm{CV}$ and renal outcome; however, the Asian subpopulation was a subgroup analysis and the sample size was not powered to detect statistical significance for the effect on each endpoint.

As with other cost-effectiveness analyses, short-term clinical trial data (with a follow-up of approx. 2.2 years) were used to make lifetime outcomes, which assumes that the risks of clinical events remain constant beyond the trial length. However, in the absence of long-term clinical follow-up data, simulation modelling is an efficient tool to forecast long-term clinical outcomes and costs of healthcare strategies. To minimise any uncertainty in long-term estimates, sensitivity and scenario analyses were performed. However, the cost-effectiveness of linagliptin should be analysed further with a longer observation period and a broader range of patients with T2D.

\section{CONCLUSIONS}

This was a cost-effectiveness analysis of linagliptin plus SoC in patients with T2D using data from the Asian subpopulation of the CARMELINA trial to calculate direct medical costs for diabetes and related $\mathrm{CV}$ and kidney events in Japan. The base case analysis showed that treatment with linagliptin plus SoC was dominant compared with SoC using the point estimates of each parameter; however, the ICER of each parameter in the one-way sensitivity analyses varied greatly and the probabilistic sensitivity analysis plot indicated a wide spread. Thus, the cost-effectiveness of linagliptin may be cautiously concluded to be favourable. However, additional analyses, which may include evaluation of real-world evidence, using a broader range of patient data over a longer duration, are still warranted.

\section{ACKNOWLEDGEMENTS}

Funding. Funding for this analysis was provided by Nippon Boehringer Ingelheim Co., Ltd. and Eli Lilly Japan K.K. The Rapid Service Fee for journal publication was also funded by Nippon Boehringer Ingelheim Co., Ltd. and Eli Lilly Japan K.K.

Medical Writing Assistance. We thank Ingrid de Ruiter, MBChB, PhD, and Sally-Anne Mitchell, PhD, of Edanz Medical Writing for providing medical writing support, which was funded by Nippon Boehringer Ingelheim Co., Ltd. and Eli Lilly Japan K.K. through EMC K.K. in accordance with Good Publication Practice 3 (GPP3) guidelines (https://www.ismpp.org/ gpp3).

Authorship. All named authors meet the International Committee of Medical Journal Editors (ICMJE) criteria for authorship for this article, take responsibility for the integrity of the work as a whole, and have given their approval for this version to be published.

Disclosures. Hirotaka Watada has received grants and/or personal fees from Astellas Pharma, Sanofi, Mitsubishi Tanabe Pharma, Novo Nordisk Pharma, Kowa, AstraZeneca, Takeda Pharmaceutical, Novartis Pharma, Nippon Boehringer Ingelheim, MSD, Sumitomo Dainippon Pharma, Eli Lilly Japan, Sanwa Kagaku Kenkyusho, Ono Pharmaceutical, Kissei Pharmaceutical, FUJIFILM Pharma, Kyowa Kirin, Daiichi Sankyo, Terumo, Pfizer Japan, Taisho Toyama Pharmaceutical and Kowa. Hiroyuki Sakamaki has received grants and/or personal fees from Eli Lilly, Nippon Boehringer Ingelheim, Chugai Pharmaceutical, Sanofi, Novo Nordisk Pharma, Astellas Pharma, Otsuka Pharmaceutical, Takeda Pharmaceutical, Kyowa Kirin, JCR Pharmaceuticals, SANDOZ, Sawai Pharmaceutical, Celltrion Healthcare, Edwards Lifesciences, Abbott Vascular, Japan Tissue Engineering and Olympus, and lecture fees from the Japan Generic Medicines Association and the Japan Biosimilar Association. Daisuke Yabe has received consulting or speaker fees 
from MSD, Novo Nordisk Pharma and Taisho Pharmaceutical, and clinically commissioned/ joint research grants from Taisho Pharmaceutical, Ono Pharmaceutical, Novo Nordisk Pharma, Arkray and Terumo. Fumiko Yamamoto and Tomoo Okamura are employees of Nippon Boehringer Ingelheim. Tatsunori Murata and Keigo Hanada are employees of CRECON Medical Assessment, which received consulting fees from Boehringer Ingelheim and Eli Lilly to support preparation of the analysis. Tetsuaki Hirase is an employee of Eli Lilly Japan.

Compliance with Ethics Guidelines. This article is based on previously conducted studies and does not contain any studies with human participants or animals performed by any of the authors.

Data Availability. The datasets generated during and/or analysed during the current study are available from the corresponding author on reasonable request.

Open Access. This article is licensed under a Creative Commons Attribution-NonCommercial 4.0 International License, which permits any non-commercial use, sharing, adaptation, distribution and reproduction in any medium or format, as long as you give appropriate credit to the original author(s) and the source, provide a link to the Creative Commons licence, and indicate if changes were made. The images or other third party material in this article are included in the article's Creative Commons licence, unless indicated otherwise in a credit line to the material. If material is not included in the article's Creative Commons licence and your intended use is not permitted by statutory regulation or exceeds the permitted use, you will need to obtain permission directly from the copyright holder. To view a copy of this licence, visit http://creativecommons.org/licenses/by$\mathrm{nc} / 4.0 /$.

\section{REFERENCES}

1. Forbes JM, Cooper ME. Mechanisms of diabetic complications. Physiol Rev. 2013;93:137-88. https://doi.org/10.1152/physrev.00045.2011.

2. International Diabetes Federation. Diabetes and cardiovascular disease. https://www.idf.org/ouractivities/care-prevention/cardiovascular-disease. html. Accessed 21 Apr 2020.

3. Nakamura J, Kamiya H, Haneda M, et al. Causes of death in Japanese patients with diabetes based on the results of a survey of 45,708 cases during 2001-2010: Report of the Committee on Causes of Death in Diabetes Mellitus. J Diabetes Investig. 2017;8:397-410. https://doi.org/10.1111/jdi.12645.

4. Haneda $M$, Noda $M$, Origasa $H$, et al. Japanese clinical practice guideline for diabetes 2016. Diabetol Int. 2018;9:1-45. https://doi.org/10.1007/ s13340-018-0345-3.

5. Haneda $M$, Noda $M$, Origasa $H$, et al. Japanese clinical practice guideline for diabetes 2016. J Diabetes Investig. 2018;9:657-97. https://doi.org/10. $1111 /$ jdi.12810.

6. Seino Y, Kuwata H, Yabe D. Incretin-based drugs for type 2 diabetes: focus on East Asian perspectives. J Diabetes Investig. 2016;7(suppl 1):102-9. https:// doi.org/10.1111/jdi.12490.

7. Rosenstock J, Perkovic V, Johansen OE, et al. Effect of linagliptin vs placebo on major cardiovascular events in adults with type 2 diabetes and high cardiovascular and renal risk: the CARMELINA randomized clinical trial. JAMA. 2019;321:69-79. https://doi.org/10.1001/jama.2018.18269.

8. Inagaki $\mathrm{N}$, Yang $\mathrm{W}$, Watada $\mathrm{H}$, et al. Linagliptin and cardiorenal outcomes in Asians with type 2 diabetes mellitus and established cardiovascular and/or kidney disease: subgroup analysis of the randomized CARMELINA ${ }^{\circledR}$ trial. Diabetol Int. 2020;11:129-41. https://doi.org/10.1007/s13340-019-00412-x.

9. Siebert U, Alagoz O, Bayoumi AM, et al. Statetransition modeling: a report of the ISPOR-SMDM Modeling Good Research Practices Task Force-3. Value Health. 2012;15:812-20. https://doi.org/10. 1016/j.jval.2012.06.014.

10. Clarke PM, Gray AM, Briggs A, et al. A model to estimate the lifetime health outcomes of patients with type 2 diabetes: The United Kingdom Prospective Diabetes Study (UKPDS) outcomes model (UKPDS no. 68). Diabetologia. 2004;47: 1747-59. https://doi.org/10.1007/s00125-0041527-z. 
11. Center for Outcomes Research and Economic Evaluation for Health, National Institute of Public Health $(\mathrm{C} 2 \mathrm{H})$. Guideline for preparing cost-effectiveness evaluation to the Central Social Insurance Medical Council. Version 2.0; approved 20 February 2019. https://c2h.niph.go.jp/tools/guideline/ guideline_en.pdf. Accessed 21 Apr 2020.

12. Kaku K, Haneda M, Sakamaki H, et al. Cost-effectiveness analysis of empagliflozin in Japan based on results from the Asian population in the EMPA-REG OUTCOME trial. Clin Ther. 2019;41:2021-40. https://doi.org/10.1016/j.clinthera.2019.07.016.

13. Sullivan PW, Ghushchyan VH. EQ-5D scores for diabetes-related comorbidities. Value Health. 2016;19:1002-8. https://doi.org/10.1016/j.jval. 2016.05.018.

14. Briggs A, Sculpher M, Claxton K. Decision modelling for health economic evaluation. Oxford: Oxford University Press; 2006.

15. Japan Ministry of Health, Labour and Welfare. Central Social Insurance Medical Council measures for the tentative introduction of cost-effectiveness assessment and major issues for institutionalization. 2017;20. (In Japanese). https://www.mhlw.go. jp/file/05-Shingikai-12404000-HokenkyokuIryouka/0000188711.pdf. Accessed 21 Apr 2020.

16. Reed SD, Li Y, Leal J, et al. Longitudinal medical resources and costs among type 2 diabetes patients participating in the Trial Evaluating Cardiovascular Outcomes with Sitagliptin (TECOS). Diabetes Obes Metab. 2018;20:1732-9. https://doi.org/10.1111/ dom.13292.

17. Govan $\mathrm{L}, \mathrm{Wu} \mathrm{O}$, Lindsay $\mathrm{R}$, et al. How do diabetes models measure up? A review of diabetes economic models and ADA guidelines. JHEOR. 2015;3: 132-52. https://doi.org/10.36469/9831.

18. Kansal A, Reifsnider OS, Proskorovsky I, et al. Costeffectiveness analysis of empagliflozin treatment in people with type 2 diabetes and established cardiovascular disease in the EMPA-REG OUTCOME trial. Diabet Med. 2019;36:1494-502. https://doi. org/10.1111/dme.14076.

19. Bosmans JE, de Bruijne MC, van Hout HP, Hermens ML, Adèr HJ, van Tulder MW. Practical guidelines for economic evaluations alongside equivalence trials. Value Health. 2008;11:251-8. https://doi.org/ 10.1111/j.1524-4733.2007.00245.x.

20. Ma RCW, Chan JCN. Type 2 diabetes in East Asians: similarities and differences with populations in Europe and the United States. Ann N Y Acad Sci. 2013;1281:64-91. https://doi.org/10.1111/nyas. 12098.

21. Tomino Y, Gohda T. The prevalence and management of diabetic nephropathy in Asia. Kidney Dis (Basel). 2015;1:52-60. https://doi.org/10.1159/ 000381757. 